\title{
Caffeic acid phenethyl ester attenuates nuclear factor-kB-mediated inflammatory responses in Müller cells and protects against retinal ganglion cell death
}

\author{
YANWEN JIA $^{1 *}$, SHENGQUN JIANG $^{2 *}$, CHEN CHEN $^{1 *}$, GUOHUA LU $^{1}$, \\ YANG XIE $^{1}$, XINCHENG SUN $^{1}$ and LIQIN HUANG ${ }^{1}$ \\ ${ }^{1}$ Eye Institute, The Affiliated Changzhou No. 2 People's Hospital of Nanjing Medical University, Changzhou, Jiangsu 213000; \\ ${ }^{2}$ Department of Ophthalmology, First Affiliated Hospital of Bengbu Medical College, Bengbu, Anhui 233000, P.R. China
}

Received August 5, 2018; Accepted April 1, 2019

DOI: $10.3892 / \mathrm{mmr} .2019 .10151$

\begin{abstract}
Glaucoma is characterized by the death of retinal ganglion cells (RGCs) and visual field defects, and is a leading cause of blindness worldwide. Caffeic acid phenethyl ester (CAPE), a natural polyphenolic found in propolis from honeybee hives, can inhibit the activation of nuclear factor $\kappa$ light-chain-enhancer of activated B cells (NF- $\mathrm{kB}$ ) and has therapeutic potential in inflammatory disease. The present study used a rat model of optic nerve crush (ONC) injury to investigate the effect of CAPE on glaucoma. The death of RGCs at day 14 was significantly reduced in CAPE-treated animals compared with the non-treated group according to Brn3a and TUNEL staining. In addition, CAPE decreased the severity of inflammation in the retina, reflected by the decreased expression of inflammatory cytokines, including interleukin (IL)-8, IL-6, inducible nitric oxide synthase, cycloooxygenase-2, tumor necrosis factor- $\alpha$ and chemokine C-C ligand-2, in CAPE-treated rats. The hypertrophy of astrocytes and Müller cells (gliosis) caused by ONC was also found to be attenuated by CAPE, accompanied by the inhibition of NF- $\mathrm{kB}$ signaling. Similarly, in vitro, CAPE suppressed the proliferation and migration of primary astrocytes induced by lipopolysaccharide, as well as the activation of NF- $\mathrm{kB}$. These results suggest that CAPE protected against RGC and attenuated inflammatory responses in a rat model of ONC by suppressing NF- $\mathrm{KB}$ activation.
\end{abstract}

Correspondence to: Dr Yanwen Jia, Eye Institute, The Affiliated Changzhou No. 2 People's Hospital of Nanjing Medical University, 29 Xinglong Lane, Changzhou, Jiangsu 213000, P.R. China

E-mail: jiayanwenvictor@126.com

*Contributed equally

Key words: caffeic acid phenethyl ester, NF-кB, inflammatory responses, Müller cells, retinal ganglion cell

\section{Introduction}

Glaucoma is a progressive neurodegenerative eye disease and is a leading cause of irreversible blindness globally (1-3). Increased intraocular pressure and retinal ganglion cell (RGC) death are the most common symptoms of glaucoma. Strategies to alleviate intraocular pressure, such as topical drugs, laser treatment and surgery, cannot completely suppress the progression of visual field defects in some patients (1). Although some neuroprotective strategies have been investigated, to date, there are no convincing data to support an effective therapy for glaucoma (4-6). Therefore, developing novel neuroprotective therapies to protect or regenerate RGCs in glaucoma is necessary.

Although the precise mechanism underlying glaucoma remains unclear, it is becoming increasingly clear that glial cell activation and neuro-inflammation exacerbate the loss of RGCs in the retina after optic nerve injury (7-9). Therefore, anti-inflammatory agents may be a potential effective neuro-protective therapy to protect RGCs in the retina of patients with glaucoma.

Recently, various natural and potentially therapeutic compounds have attracted the attention of researchers (10-12). Caffeic acid phenethyl ester (CAPE), an active phenolic component in the propolis of the honeybee hive, has been reported to be a potent antioxidant and suppressor of nuclear factor $\kappa$-light-chain-enhancer of activated B cells (NF- $\kappa \mathrm{B})$ (13). CAPE has a multitude of beneficial biological properties, including antioxidant, anti-inflammatory, antiviral, anti-proliferative, neuroprotective, hepatoprotective and cardioprotective capacities (13-18). Some studies have described the anti-apoptosis effect of CAPE in neurons induced by ischemia-reperfusion or low potassium levels by blocking the production of reactive oxygen species and inhibiting caspase activity $(19,20)$. However, there have been no published studies investigating the role of CAPE in protecting against RGC death using the optic nerve crush (ONC) model for glaucoma.

In the present study, the survival and apoptosis of RGCs after ONC injury with and without CAPE treatment were compared. The protective role of CAPE in ONC-induced RGC apoptosis and neuro-inflammation was demonstrated. The 
expression of cytokines in the retina was suppressed by CAPE, as well as the activation of NF- $\kappa \mathrm{B}$ in astrocytes. The present study provides detailed evidence supporting the neuroprotective effect of CAPE on RGCs.

\section{Materials and methods}

Animals and the ONC model. All 72 Sprague-Dawley rats (8-12 weeks of age, male; weight, 170-200 g) used in this study were provided by the Shanghai Laboratory Animal Center of the Chinese Academy of Sciences (Shanghai, China). Animals were housed in a temperature-controlled room $\left(22 \pm 3^{\circ} \mathrm{C}\right)$ with a 12-h light/dark cycle under specific-pathogen free conditions and provided with free access to water and food. All experimental animal protocols were approved by the Institutional Animal Care and Use Committee of Wenzhou Medical University (Wenzhou, Zhejiang, China). The ONC protocol was adapted from previous studies $(10,21)$. Briefly, rats were anesthetized using pentobarbital sodium $(40 \mathrm{mg} / \mathrm{kg}$; Sigma-Aldrich; Merck KGaA, Darmstadt, Germany). A small incision was then made in the superior and lateral conjunctiva, and gentle dissection was performed to expose the optic nerve, and to avoid tissue damage and infraorbital trauma. The optic nerve was crushed for $10 \mathrm{sec}$ using a vascular clip at the site $2 \mathrm{~mm}$ posterior to the globe. The integrity of the retinal blood supply was verified, and rats with severely reduced perfusion were excluded. Before the application of the coverslip, the eye was moisturized using sodium hyaluronate $1 \mathrm{mg} / \mathrm{ml}$ (Hylo-COMOD, Ursapharm, Germany). For each experiment, ONC was performed on the left eye.

CAPE treatment. Rats were randomly assigned to one of four groups ( $\mathrm{n}=18$ each): the control (Con); the Con + CAPE; the ONC; and the ONC + CAPE. No surgery was performed in the control rats. Rats were injected with $10 \mu \mathrm{mol} / \mathrm{kg}$ CAPE (Sigma-Aldrich; Merck KGaA) intraperitoneally 10 min after the surgery according to a method described in a previous study (22). Equal amounts of vehicle (i.e., instead of CAPE) were administered to the rats in the control and the ONC groups.

Brn3a-labeled flat-mounted retinas. Fourteen days after surgery, the rats were euthanized with carbon dioxide, and the eyeballs were enucleated and fixed using $4 \%$ paraformaldehyde at $4{ }^{\circ} \mathrm{C}$ overnight. Retina flat mounts were carefully prepared and incubated with blocking buffer $(0.3 \%$ Triton X-100 and $2 \%$ donkey serum) for $1 \mathrm{~h}$ at room temperature after washing with phosphate-buffered saline (PBS). The retina flat mounts were then incubated with rabbit monoclonal antibody for brain-specific homeobox/POU domain protein 3A (Brn-3a) (dilution 1:100; cat. no. ab232480; Abcam, Cambridge, MA, USA) overnight at $4^{\circ} \mathrm{C}$, followed by anti-rabbit Alexa Fluor 594 (dilution 1:500; cat. no. R37117; Thermo Fisher Scientific, Inc., Waltham, MA, USA) for $2 \mathrm{~h}$ at room temperature. The slides were then examined using a confocal microscope (TCS SP5; Leica Microsystems GmbH, Wetzlar, Germany; magnification, $\mathrm{x} 200$ ), and 10 microphotographs were captured for each retina.

TUNEL apoptosis assay. Terminal deoxynucleotidyl transferase dUTP nick end labeling (TUNEL) staining was performed using a commercially available kit (One-Step TUNEL Apoptosis Assay kit; cat. no. C1086; Beyotime Institute of Biotechnology, Haimen, China). Frozen tissue sections were rinsed with PBS for $10 \mathrm{~min}$ and incubated with $0.5 \%$ Triton X-100 for $5 \mathrm{~min}$ at room temperature. The TUNEL reaction mixture (50 $\mu 1$ for each section) was added and stained for $60 \mathrm{~min}$ at $37^{\circ} \mathrm{C}$. DAPI was also used to visualize nuclei. TUNEL-positive cells were examined using a confocal microscope, three random fields for each sample was captured.

Immunofluorescent staining. Retinas were harvested on day 14 after ONC, and frozen sections ( $8 \mu \mathrm{m}$ thick) were prepared using a microtome (Leica Microsystems $\mathrm{GmbH}$ ). Sections were blocked with PBS containing $0.3 \%$ Triton X-100, $1 \%$ BSA and donkey serum for $2 \mathrm{~h}$ to avoid non-specific staining. After blocking, the sections were stained with primary antibodies for mouse monoclonal anti-glial fibrillary acidic protein (GFAP) (dilution 1:200; cat. no. ab10062) and rabbit polyclonal anti-NF-кB-P65 (dilution 1:100; cat. no. ab16502) (both from Abcam) at $4^{\circ} \mathrm{C}$ overnight, and further stained with fluorescein conjugated secondary antibodies (anti-mouse Alexa Fluor 488 (dilution 1:500; cat. no. R37120; Thermo Fisher Scientific, Inc.) and anti-rabbit Alexa Fluor 594). Sections were sealed and examined using a scanning confocal microscope (Leica Microsystems $\mathrm{GmbH}$ ).

Rat primary astrocyte culture and cell treatment. Primary rat astrocytes were prepared according to methods described in previous studies $(23,24)$. Briefly, mixed-glia cultures were prepared from the dissected brains of newborn Sprague-Dawley rats (1-3 days) and cultured in Dulbecco's modified Eagle's medium (DMEM)/F12 supplemented with $10 \%$ fetal bovine serum (FBS). The culture medium was changed on days 3 and 6 . For purification, oligodendrocytes and microglia were removed by shaking the flasks at $200 \mathrm{rpm}$ for $2 \mathrm{~h}$ at $37^{\circ} \mathrm{C}$ (days 10-12). The isolated astrocytes were confirmed by immunostaining for CD11b and GFAP; astrocyte purity was $>95 \%$. The second passage of astrocytes were used in this study.

Astrocyte migration assay. Astrocytes were seeded in 24-well plates. Astrocyte monolayers were gently and perpendicularly scratched using a sterile pipette tip across the well when the cells reached $70-80 \%$ confluence. The wells were washed twice with medium to remove the detached cells and treated with either CAPE or together with lipopolysaccharide (LPS) for $16 \mathrm{~h}$ before being examined by inverted phase contrast microscope. Photomicrographs of five randomly chosen fields were captured, and the gap distance was quantitatively evaluated using ImageJ v1.8.0 software (NIH; National Institutes of Health, Bethesda, MD, USA). The migration rate was obtained by comparing the migration distance of the experimental groups with the control group.

Cell viability assay. Astrocyte viability was measured using a commercially available Cell Counting Kit-8 (CCK-8; Dojindo Laboratories, Kumamoto, Japan). Astrocytes were plated in 96-well plates $\left(5 \times 10^{3}\right.$ cells/well $)$ and treated with different concentrations of CAPE $(0,1,5,10,20$ or $40 \mu \mathrm{M})$ or LPS $(1 \mu \mathrm{g} / \mathrm{ml})$ for $48 \mathrm{~h}$. To measure astrocyte viability, $10 \mu \mathrm{l}$ of 
CCK-8 solution was added to each well in the dark. After a 1-h incubation at $37^{\circ} \mathrm{C}$, absorbance was read using a microplate reader (BioTek Instruments, Inc., Winooski, VT, USA) at $450 \mathrm{~nm}$.

Quantitative polymerase chain reaction ( $P C R$ ). RNA from retinas and astrocytes was isolated using a commercially available kit (miRNeasy Mini kit; Qiagen, Duesseldorf, Germany) and complementary DNA was synthesized using PrimeScript $^{\mathrm{TM}}$ RT reagent kit with gDNA Eraser (Takara Biotechnology Co., Ltd., Dalian, China). Real-time quantitative PCR was performed using SYBR Premix Ex Taq ${ }^{\mathrm{TM}}$ II kit (Takara Biotechnology Co., Ltd.). Messenger RNA (mRNA) expression of interleukin (IL)-8, IL-6, inducible nitric oxide synthase (iNOS), cyclooxygenase-2 (COX-2), tumor necrosis factor- $\alpha$ (TNF- $\alpha$ ) and C-C motif ligand-2 (CCL-2) in retinas were normalized to $\beta$-actin using the $2^{-\Delta \Delta \mathrm{Cq}}$ method (25).

Western blot analysis. Retinas were carefully separated from the eye balls, placed in lysis buffer (1X RIPA Buffer, cat. no. 9806S; Cell Signaling Technology, Danvers, MA, USA) and ultrasonicated on ice. Cell lysates were centrifuged at 12,000 x g for $10 \mathrm{~min}$ at $4^{\circ} \mathrm{C}$ to collect the supernatant protein. Protein concentrations were determined using a Pierce BCA Protein Assay Kit (cat. no. 23225; Thermo Fisher Scientific, Inc.) and $30 \mu \mathrm{g}$ of each sample was blotted with $10 \%$ SDS-polyacrylamide gel electrophoresis and transferred on to polyvinylidene difluoride (PVDF) membranes (EMD Millipore, Billerica, MA, USA). Following blocking with $5 \%$ nonfat milk at room temperature $\left(20-25^{\circ} \mathrm{C}\right)$ for $2 \mathrm{~h}$, the membranes were incubated with monoclonal anti-GFAP (dilution 1:1,000; cat. no. ab10062), polyclonal anti-NF- $\mathrm{B}-\mathrm{P} 65$ (dilution 1:1,000; cat. no. ab16502) or monoclonal anti- $\beta$-actin (dilution 1:1,000; cat. no. ab8226) (all from Abcam) for $18 \mathrm{~h}$ at $4^{\circ} \mathrm{C}$. Protein expression was determined using horseradish peroxidase-conjugated secondary antibodies (dilution 1:2,000; cat. nos. 7074 and 7076; Cell Signaling Technology) and visualized using SuperSignal $^{\mathrm{TM}}$ West Femto Substrate Trial kit (Thermo Fisher Scientific, Inc.). ImageJ v1.8.0 (National Institutes of Health) was used for densitometry.

Statistical analysis. All data in the present study are expressed as mean \pm standard deviation (SD) and analyzed using GraphPad Prism 6.0 (GraphPad Software Inc., La Jolla, CA, USA). Two-way analysis of variance (ANOVA), followed by Turkey's multiple comparison test, was used to compare the different groups; $\mathrm{P}<0.05$ was considered to indicate a statistically significant result.

\section{Results}

CAPE suppresses the loss of RGCs after ONC. To investigate the protective effects of CAPE against ONC-induced retinal damage, Brn3a immunofluorescence staining was performed on the retinal flat mounts 14 days after ONC injury. Representative retinal images are shown in Fig. 1A. Brn3a-labeled RGCs were counted at the same distance from the optic nerve head. As expected, the number of Brn3a-stained RGCs decreased in the vehicle-treated ONC group $(\mathrm{P}<0.001)$ compared with the control group. Notably, CAPE significantly suppressed the loss of RGCs compared with the vehicle-treated
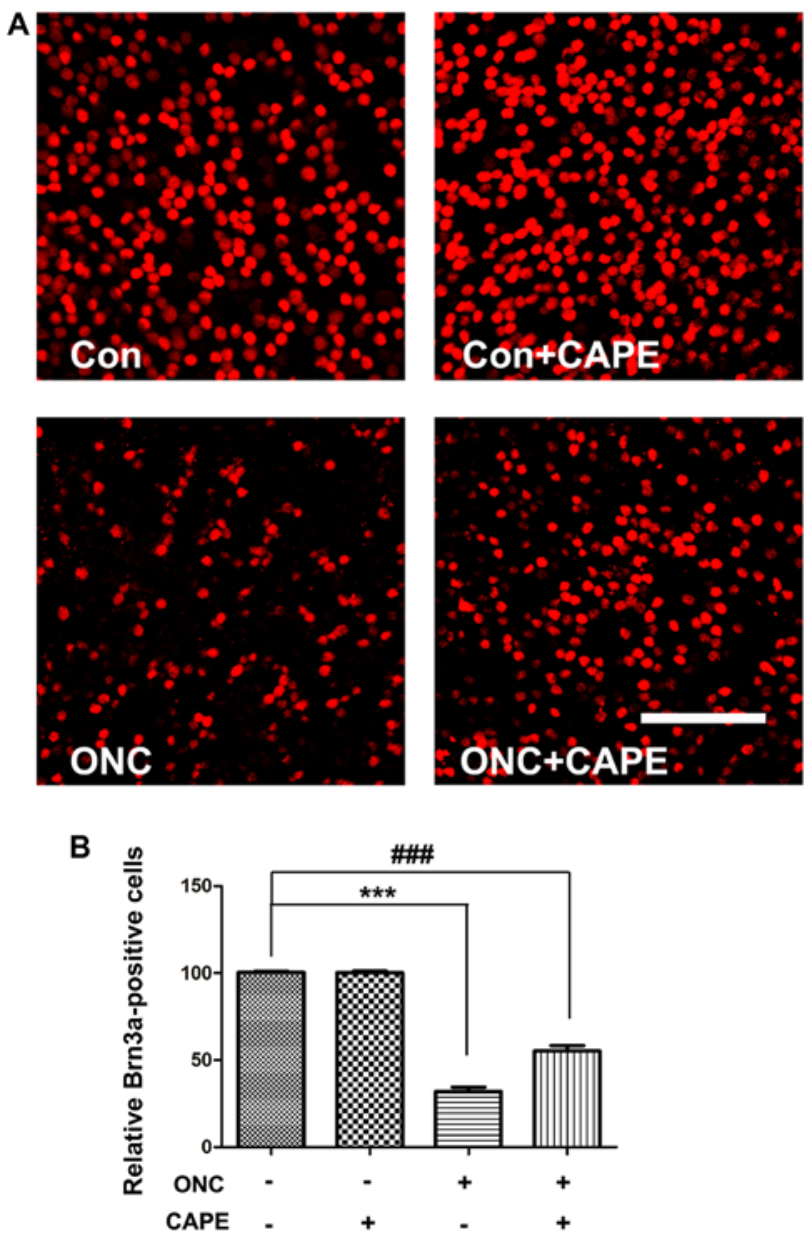

Figure 1. CAPE enhances RGC survival in the retina after ONC. Immunostaining of Brn3a (red) was performed to determine the survival of RGCs in retinas at day 14 after ONC. (A) Representative immunostaining images of retinas. Scale bar, $100 \mu \mathrm{m}$. (B) Analysis of the number of Brn3a-positive RGCs in the different groups. (mean $\pm \mathrm{SD}, \mathrm{n}=6$; ${ }^{* * *} \mathrm{P}<0.001$ compared with the control group; ${ }^{\# \# \# P<0.001 ~ c o m p a r e d ~ w i t h ~ t h e ~ O N C ~ g r o u p) . ~ C A P E, ~ c a f f e i c ~}$ acid phenethyl ester; RGCs, retinal ganglion cells; ONC, optic nerve crush.

ONC group (Fig. 1B), but demonstrated no effect on RGCs in intact retinas.

$C A P E$ attenuates the apoptosis of $R G C s$ in retinas after $O N C$. To further examine the protective role of CAPE in the retina after ONC, TUNEL-positive apoptotic cells in retina frozen sections were stained at day 7. Few apoptotic cells in the retinas were observed in the Con group and Con+CAPE group (Fig. 2A), and a large number of TUNEL-positive cells were found in the retinas after ONC. However, fewer TUNEL-stained cells were found in the ONC+CAPE group compared with the ONC group (Fig. 2B). These results confirmed the protective effect of CAPE in the retina, and CAPE significantly attenuated RGC apoptosis induced by ONC.

CAPE inhibits cytokine expression in retinas after $O N C$. To determine whether the inflammatory response caused by ONC injury is affected by CAPE treatment, cytokine expression in retinas from the different groups was assessed using real-time PCR. Compared with the control group, mRNA levels of IL-8 (Fig. 3A), IL-6 (Fig. 3B), iNOS (Fig. 3C), 
A
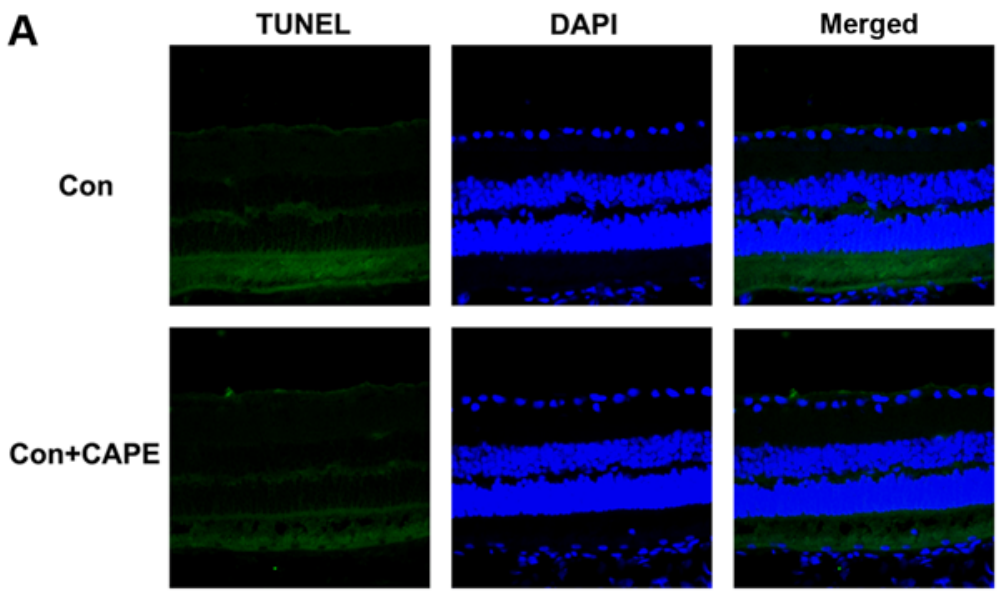

B
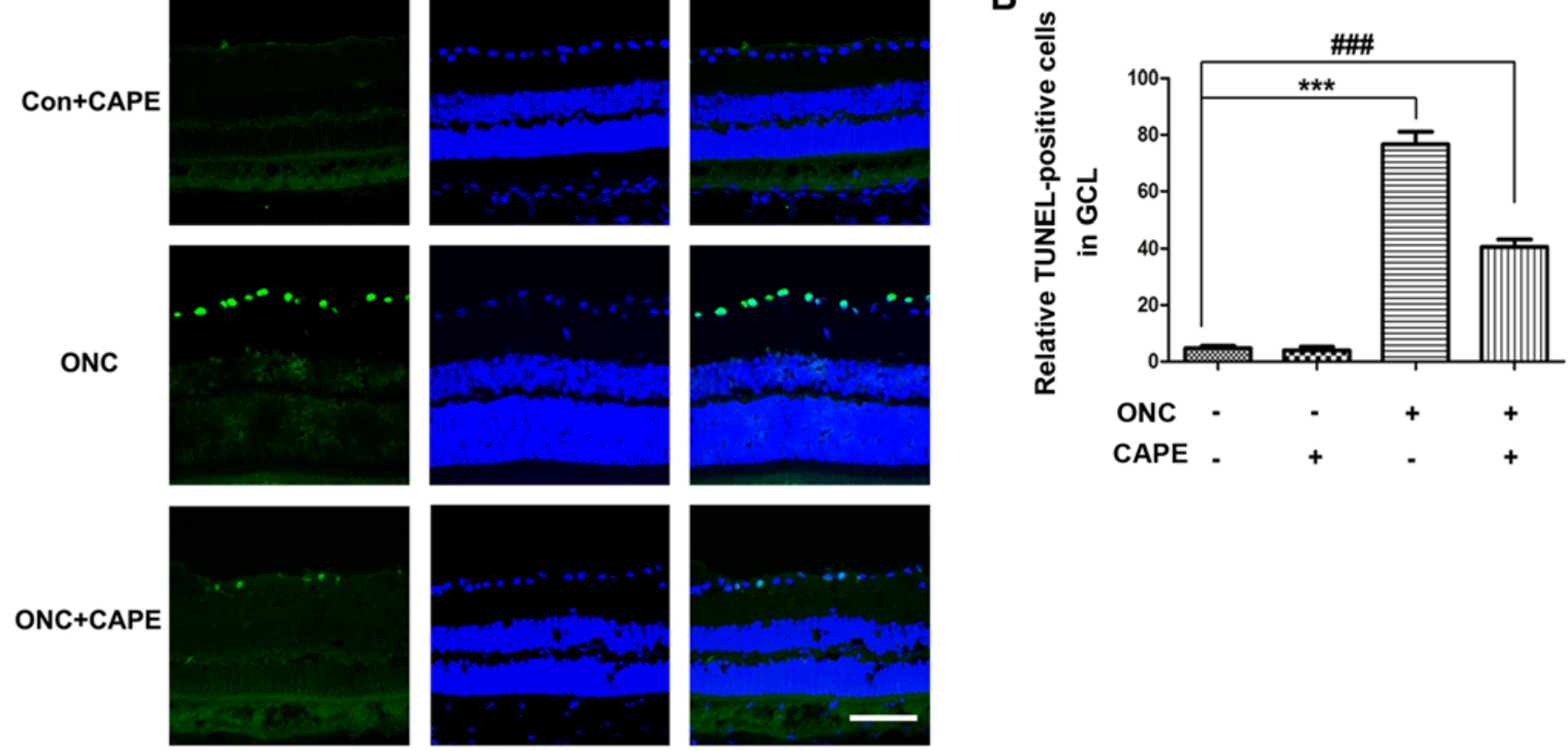

Figure 2. CAPE decreases the death of RGCs in the retina. (A) TUNEL staining of retinal sections at day 7 post ONC. TUNEL (green) and DAPI (blue) were double stained. Scale bar, $100 \mu \mathrm{m}$. (B) Analysis of the ratio of TUNEL-positive RGCs in retinas from the different groups. (mean $\pm \mathrm{SD}, \mathrm{n}=6,{ }^{* * *} \mathrm{P}<0.001$ compared with the control group; \#\#" $\mathrm{P}<0.001$ compared with the ONC group). CAPE, caffeic acid phenethyl ester; RGCs, retinal ganglion cells; ONC, optic nerve crush; TUNEL, terminal deoxynucleotidyl transferase dUTP nick end labeling.

COX-2 (Fig. 3D), TNF- $\alpha$ (Fig. 3E) and CCL-2 (Fig. 3F), were significantly upregulated in the ONC group. Expression of these pro-inflammatory cytokines were suppressed in rats that received CAPE after ONC injury (Fig. 3A-F). These results suggest that CAPE could effectively inhibit inflammation after ONC.

CAPE prevents gliosis caused by ONC injury by inhibiting $N F-\kappa B$ activation. It is well established that astrocyte hypertrophy and Müller cells (gliosis) are induced by ONC (26). To examine the contribution of activated astrocytes to the protective role of CAPE after ONC injury, the expression of GFAP by western blot analysis and immunostaining of retinal sections was examined. As expected, the expression of GFAP was increased on day 7 after ONC (Fig. 4A and B) and mainly localized in astrocytes (Fig. 4D). The protein level of GFAP was lower in the retinas of rats treated with CAPE than that in the ONC group (Fig. 4A and B). Additionally, the increase in NF- $\kappa B-p 65$ in the retina caused by ONC injury was suppressed by CAPE (Fig. 4A and C). Importantly, double immunostaining revealed that NF- $\kappa \mathrm{B}-\mathrm{p} 65$ was mainly expressed in GFAP-positive cells (Fig. 4D), suggesting that CAPE may attenuate the gliosis response by inhibiting NF- $\mathrm{KB}$ activation.

CAPE suppresses the proliferation and migration of astrocytes induced by LPS. To explore the effect of CAPE on astrocytes, rat primary astrocytes were prepared and treated with different concentrations of CAPE in vitro. Low doses of CAPE $(0,1$, $5,10$ or $20 \mu \mathrm{M})$ demonstrated no effect on the viability of astrocytes; however, $40 \mu \mathrm{M}$ CAPE decreased the viability of astrocytes after a 48-h treatment (Fig. 5A). LPS stimulated the proliferation of astrocytes, indicated by the increase in cell viability (Fig. 5B). Notably, CAPE (5, 10 or $20 \mu \mathrm{M})$ inhibited the proliferation of astrocytes induced by LPS, reflected by the significant decrease in cell viability (Fig. 5B). Furthermore, CAPE suppressed the migration of astrocytes induced by LPS (Fig. 5C and D). Together, these results suggested that the protective role of CAPE in ONC may be associated with its effect on astrocyte proliferation and migration.

CAPE suppresses the expression of pro-inflammatory cytokines and the activation of $N F-\kappa B$ in astrocytes. To investigate whether the effect of CAPE on astrocytes was relevant to its protective function in $\mathrm{ONC}$, the expression of pro-inflammatory cytokines and the activation of NF- $\mathrm{KB}$ in primary astrocytes were examined. As expected, the increase in IL-8, IL-6, iNOS, COX-2, TNF- $\alpha$ and CCL-2 (Fig. 6A-F) in astrocytes were highly suppressed by CAPE treatment. Similarly, the activation of NF- $\mathrm{KB}$ was significantly suppressed by CAPE, demonstrated by protein expression of GFAP and NF-kB-p65 (Fig. 6G-I). These data were consistent with the results in the ONC model animals. 
A

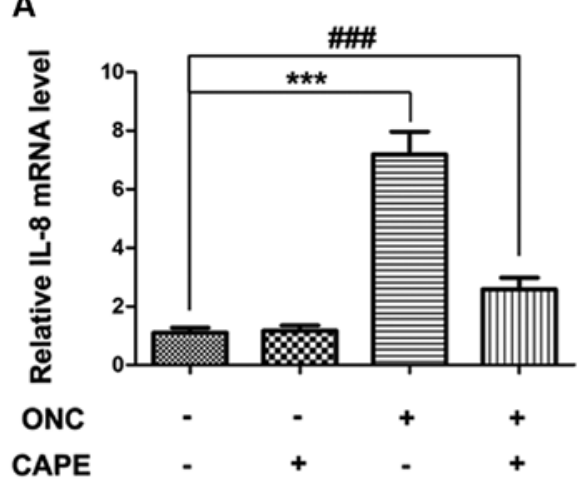

C

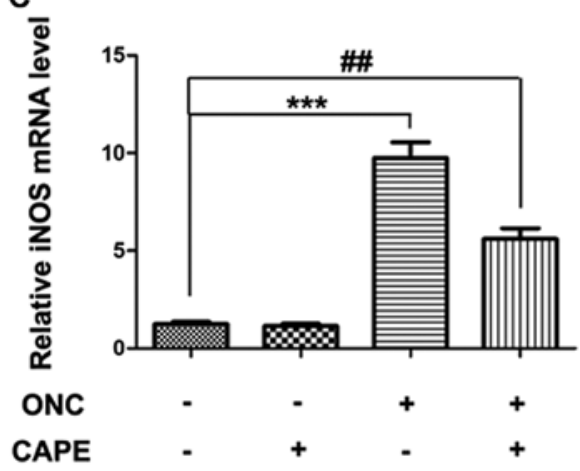

E

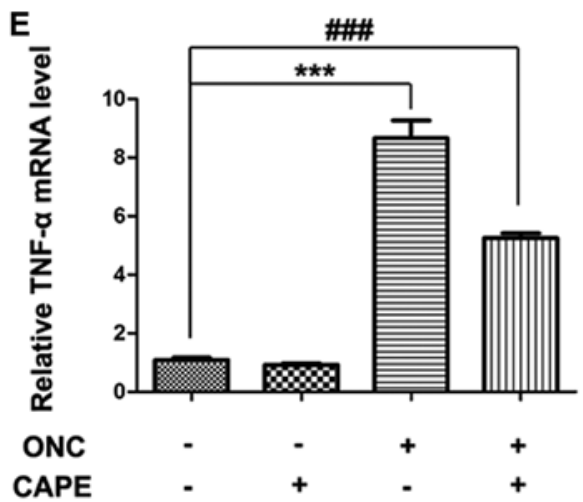

B

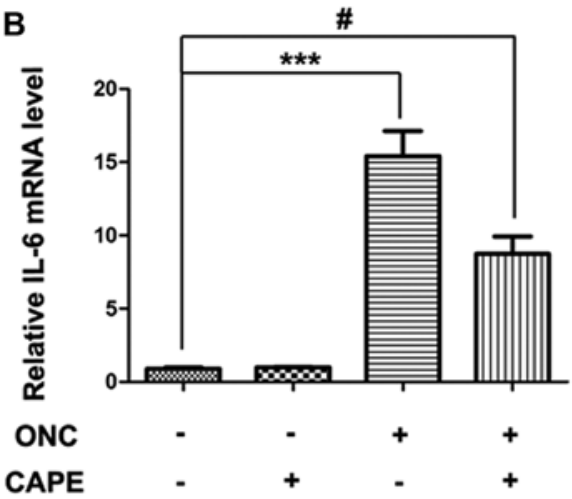

D

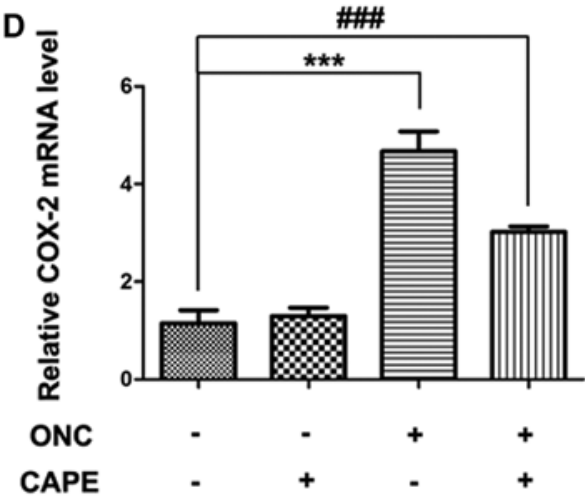

$F$

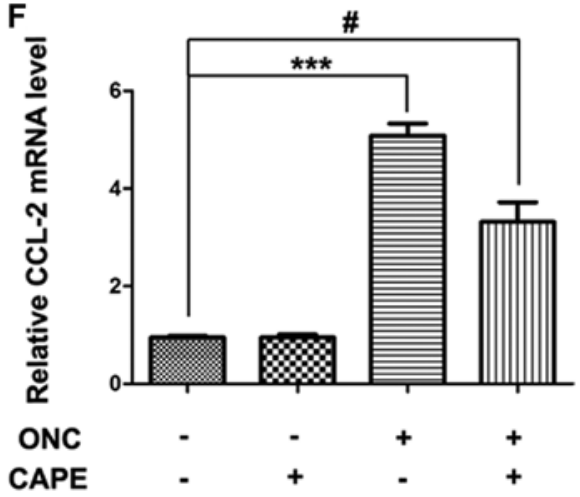

Figure 3. Influence of CAPE on pro-inflammatory cytokines following ONC. Relative mRNA levels of (A) IL-8, (B) IL-6, (C) iNOS, (D) COX-2, (E) TNF- $\alpha$ and (F) CCL-2 in retinas 7 days after ONC were determined by quantitative PCR. (mean $\pm \mathrm{SD}, \mathrm{n}=6,{ }^{* * *} \mathrm{P}<0.001$ compared with the control group; ${ }^{*} \mathrm{P}<0.05$, ${ }^{\# \#} \mathrm{P}<0.01,{ }^{\# \#} \mathrm{P}<0.001$ compared with the ONC group). CAPE, caffeic acid phenethyl ester; ONC, optic nerve crush; IL-8, interleukin (IL)-8; iNOS, inducible nitric oxide synthase; COX-2, cyclooxygenase- 2 ; TNF- $\alpha$, tumor necrosis factor- $\alpha$; CCL-2, C-C motif ligand.

\section{Discussion}

Several animal models have been developed to study glaucoma (27-29) among which optic nerve crush (ONC) is widely used to evaluate the survival of injured retinal ganglion cells (RGCs) and inflammation of glia. ONC is an acute injury that kills the majority of RGCs within the first 2 weeks and triggers atypical inflammatory response within the injured eye (30-33). In the present study, using a rat ONC model, we have demonstrated for the first time that caffeic acid phenethyl ester (CAPE), exerted a neuroprotective role and attenuated inflammatory responses by inhibiting nuclear factor kappa light-chain-enhancer of activated $\mathrm{B}$ cells $(\mathrm{NF}-\kappa \mathrm{B})$ activation.

RGC death is an important feature of glaucoma, which can lead to irreversible vision loss. CAPE markedly attenuated the symptoms of ONC, indicated by the increase in Brn3a-labeled RGCs and the decrease in TUNNEL-positive apoptotic RGCs in the retinas of rats that received CAPE treatment $10 \mathrm{~min}$ after surgery. In agreement with these data, Shi and colleagues reported that CAPE inhibited the apoptosis of retinal cells after ischemia-reperfusion injury (22).

$N F-\kappa B$ signaling is always activated in response to elevated intraocular pressure, vascular diseases and oxidative stress. Oxidative stress has been reported to be largely responsible for the loss of RGCs, molecular damage and cellular dysfunction in glaucoma. NF- $\kappa \mathrm{B}$ activation is a common pathological pathway in many diseases characterized by inflammation, such as rheumatoid arthritis, type I diabetes and glaucoma (34-37). NF- $\kappa \mathrm{B}$ activation was reported in both human glaucoma optic nerve head astrocytes and in experimental animal models $(38,39)$. In this study, 

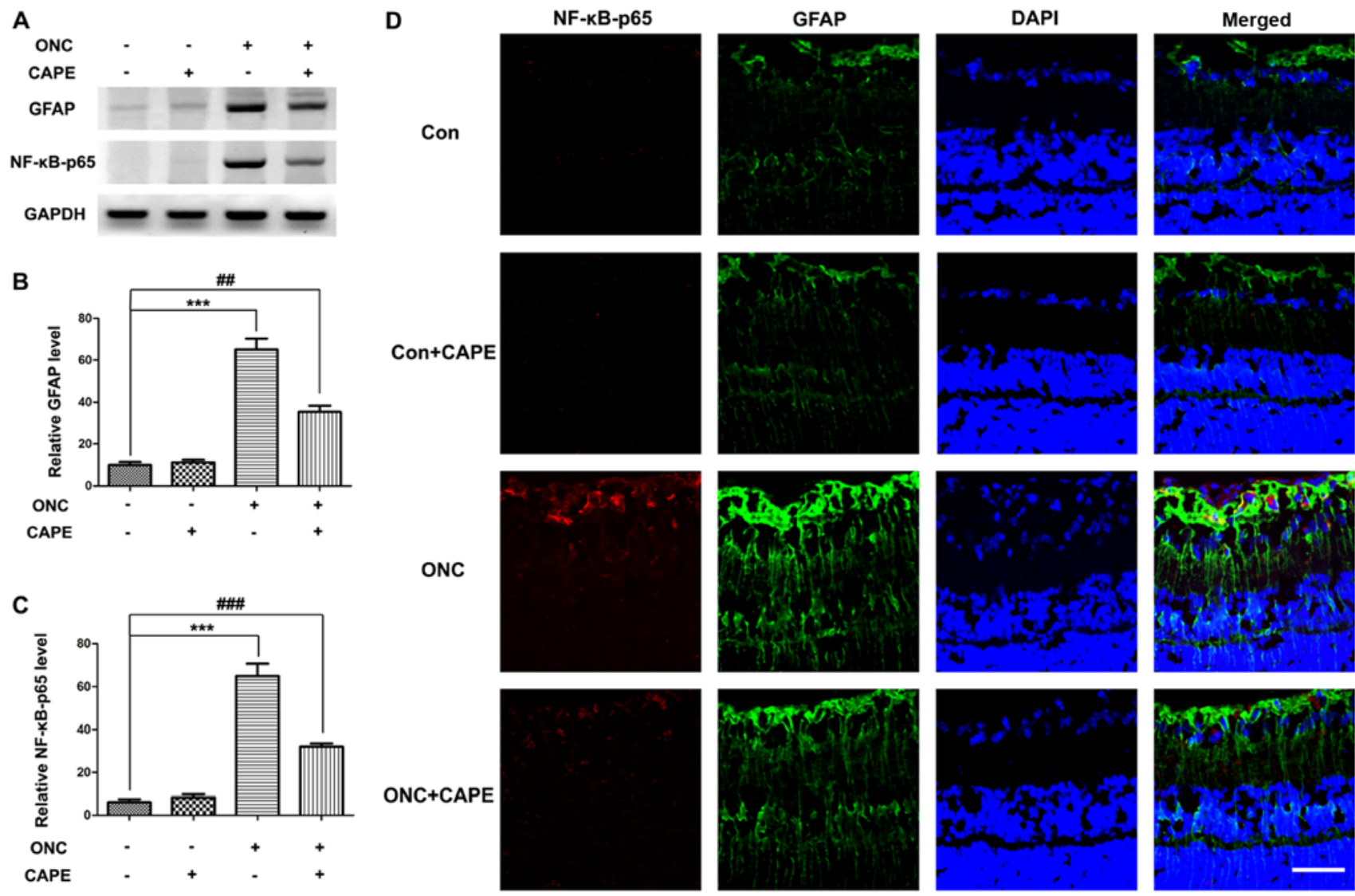

Figure 4. Effect of CAPE on the activation of NF- $\mathrm{kB}$ in astrocytes. On day 7 after ONC, rat retinas in the indicated groups were harvested and lysed or subjected to frozen sections. (A) Protein levels of GFAP and NF- $\mathrm{kB}-\mathrm{p} 65$ in retina were detected by western blot analysis. Expression of GFAP (B) and NF- $\mathrm{KB}-\mathrm{p} 65(\mathrm{C})$ in the different groups was compared using densitometric analysis. (mean $\pm \mathrm{SD}, \mathrm{n}=6{ }^{* * * *} \mathrm{P}<0.001$ compared with the control group; ${ }^{\# \#} \mathrm{P}<0.01$,

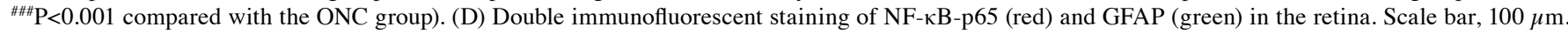
CAPE, caffeic acid phenethyl ester; ONC, optic nerve crush; GFAP, anti-glial fibrillary acidic protein.

\section{A}

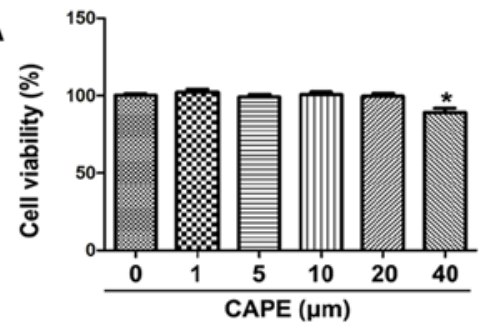

C Con

$\mathbf{O h}$

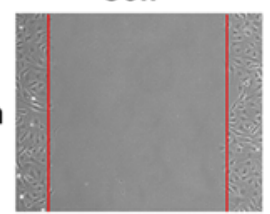

$24 \mathrm{~h}$

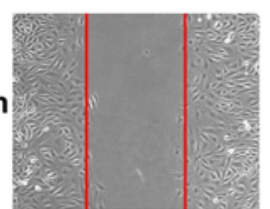

\section{Con+CAPE}
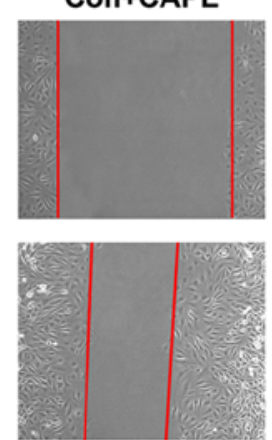
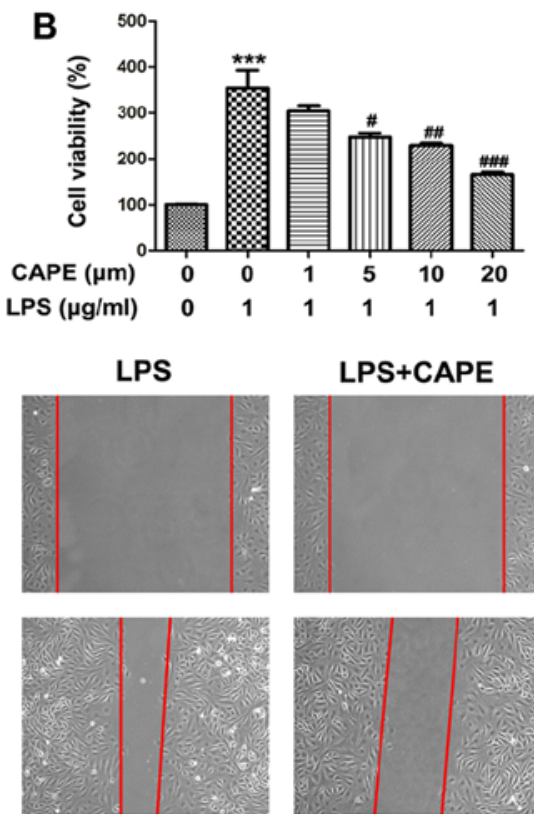

\section{LPS+CAPE}
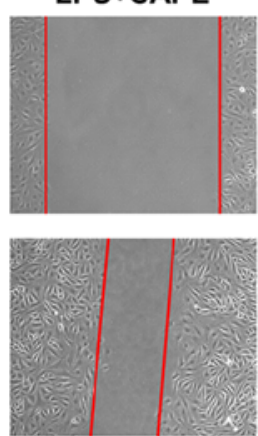

D

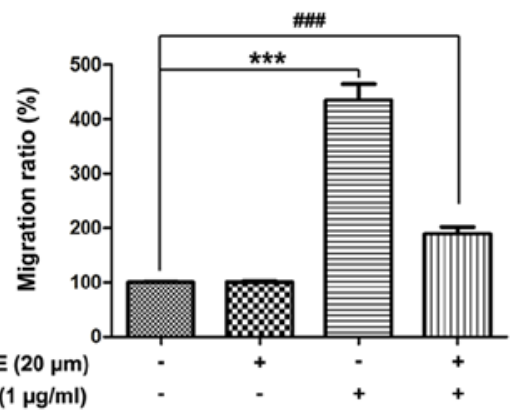

Figure 5. Effect of CAPE on the viability and migration of primary astrocytes. (A) Cell viability of astrocytes exposed to different concentrations of CAPE $(0,1,5,10,20$ or $40 \mu \mathrm{M})$ for $48 \mathrm{~h}$ were detected with CCK-8. (B) The effect of different doses of CAPE $(0,1,5,10$ or $20 \mu \mathrm{M})$ on the cell viability of LPS-treated astrocytes. (C) Migration of astrocytes treated with LPS or CAPE were examined by scratch assay. No difference was found between the Con and Con+CAPE groups $24 \mathrm{~h}$ after placing the scratch. An obvious increase in astrocytes in the LPS group was found to have migrated into the denuded space when compared to the Con group. CAPE significantly suppressed the migration of the astrocytes treated with LPS. (D) Migration ratio (\%) of astrocytes. $\left(\mathrm{n}=5,{ }^{*} \mathrm{P}<0.05,{ }^{* * * *} \mathrm{P}<0.001\right.$ compared with the Con group; ${ }^{\#} \mathrm{P}<0.05,{ }^{\# \#} \mathrm{P}<0.01,{ }^{\# \# \#} \mathrm{P}<0.001$ compared with the LPS group). CAPE, caffeic acid phenethyl ester; CCK-8, Cell Counting Kit-8; LPS, lipopolysaccharide. 
A
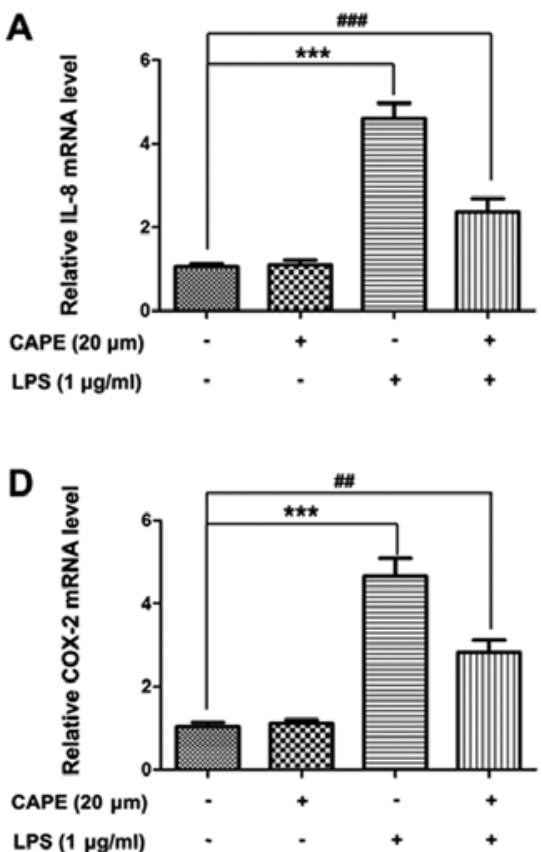

G

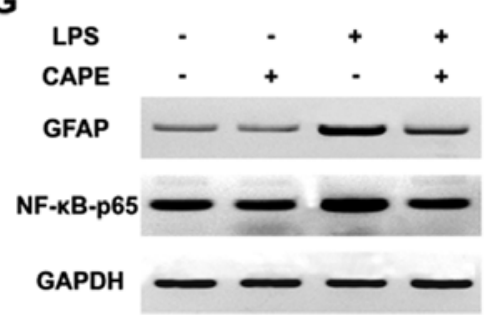

B

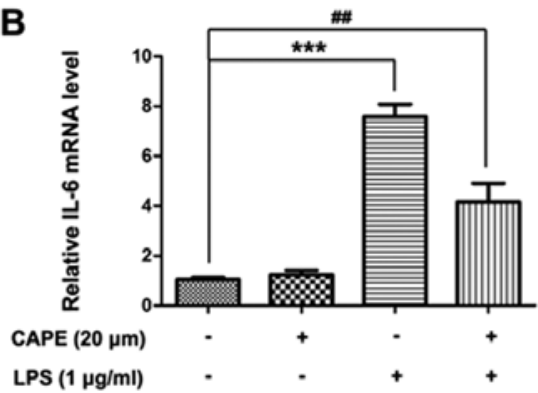

E

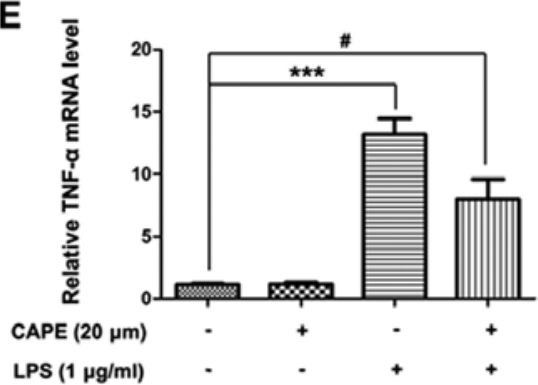

H

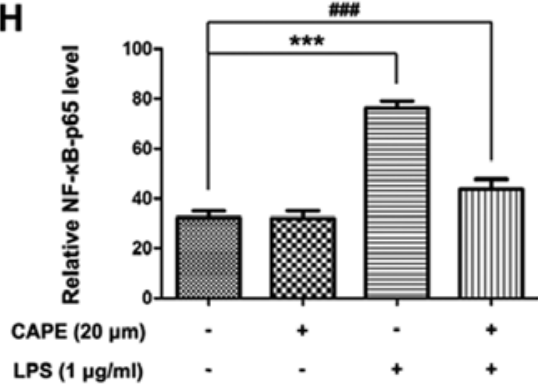

C

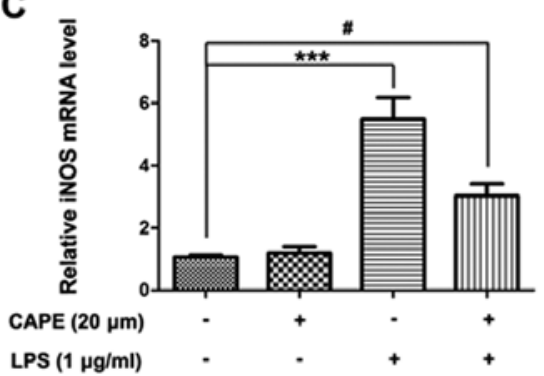

F

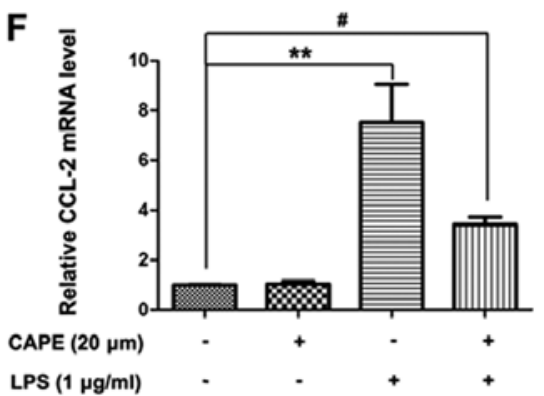

I

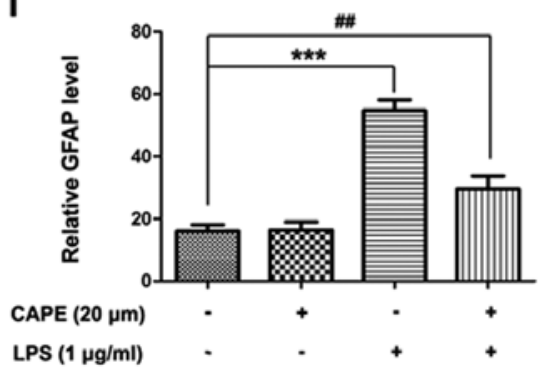

Figure 6. CAPE suppresses the activation of astrocytes. Astrocytes were exposed to CAPE $(20 \mu \mathrm{M})$ or LPS $(1 \mu \mathrm{g} / \mathrm{ml})$ for $12 \mathrm{~h}$. Total RNA of astrocytes from the different groups was extracted and the relative mRNA levels of (A) IL-8, (B) IL-6, (C) iNOS, (D) COX-2, (E) TNF- $\alpha$ and (F) CCL-2 in astrocytes were examined quantitatively using real-time quantitative PCR. (G) Protein expression of GFAP and NF-kB-p65 in astrocytes treated with or without CAPE and LPS in vitro was assessed by western blot analysis. The protein level of (H) GFAP and (I) NF-kB-p65 in the different groups was evaluated by densitometric analysis. (mean $\pm \mathrm{SD}, \mathrm{n}=3,{ }^{* *} \mathrm{P}<0.01,{ }^{* * *} \mathrm{P}<0.001$ compared with the control group; ${ }^{\# \mathrm{P}}<0.05,{ }^{\# \#} \mathrm{P}<0.01,{ }^{\# \# \# "} \mathrm{P}<0.001$ compared with the LPS group). CAPE, caffeic acid phenethyl ester; LPS, lipopolysaccharide; IL-8, interleukin (IL)-8; iNOS, inducible nitric oxide synthase; COX-2, cyclooxygenase-2; TNF- $\alpha$, tumor necrosis factor- $\alpha$; CCL-2, C-C motif ligand.

activation of NF- $\mathrm{kB}$ in astrocytes was also induced in a rat ONC model and was suppressed in CAPE-treated animals. Activated NF- $\mathrm{KB}$ enters the nucleus to induce transcription of downstream inflammatory cytokines that exacerbate oxidative stress. We found that CAPE also reduced the expression of cytokines interleukin (IL)-8, IL-6, inducible nitric oxide synthase (iNOS), cyclooxygenase-2 (COX-2), tumor necrosis factor- $\alpha$ (TNF- $\alpha$ ) and C-C motif ligand-2 (CCL-2), which was consistent with previous studies in experimental ulcerative colitis (40) and acute spinal cord injury (41). These data suggest that the protective effects of CAPE in ONC may be related to the alleviation of neuro-inflammation in the retina.

Considering the decisive role of gliosis in the pathological course of RGC damage $(42,43)$ we analyzed the expression of GFAP using western blot analysis and immunohistochemical staining. As expected, GFAP in the retina was upregulated after ONC injury. It was interesting to find that CAPE alleviated gliosis caused by ONC, as evidenced by the significant downregulation of GFAP and NF- $\kappa \mathrm{B}$ compared with the ONC group. The effect of CAPE in astrocytes was further confirmed in in vitro experiments. CAPE suppressed reactive astrogliosis induced by LPS in vitro, reflected by the inhibition of proliferation and migration of primary cultured astrocytes, as well as the expression of GFAP and NF- $\mathrm{kB}$.

Data from the present study indicated that the protective role of CAPE in experimental glaucoma may be mediated by the anti-inflammatory function of CAPE in astrocytes through inhibition of NF- $\kappa B$ activation. This may not be the only protective role of CAPE; other pathological changes in glaucoma, including mitochondrial damage, endothelial dysregulation and hypoxia may also be influenced by CAPE treatment. We speculate that CAPE possibly functions by regulating intracellular or extracellular signals, such as reactive oxygen species, which are massively generated in glaucoma-related oxidative stress and lead to the activation of NF- $\mathrm{BB}(37,44)$. Further studies are necessary to explore the molecular mechanisms by which CAPE inhibits activation of NF- $\kappa$ B. Whether CAPE can block the translocation of NF- $\mathrm{KB}$ to the nucleus and inhibit $\mathrm{NF}-\kappa \mathrm{B}$ binding to the target sequence warrants further investigation. 
In conclusion, our findings suggest that systemic administration of CAPE appears to afford neuroprotection in a rat model of ONC injury, and controls retinal gliosis and the release of pro-inflammatory mediators by suppressing $\mathrm{NF}-\kappa \mathrm{B}$ activation. CAPE may be a promising candidate for the treatment of glaucoma. However, CAPE may act differently in mice, rats or humans; therefore, more in-depth, systematic research is needed to determine the molecular mechanisms, optimal concentrations, and possible side effects of CAPE before its clinical application.

\section{Acknowledgements}

Not applicable.

\section{Funding}

No funding was received.

\section{Availability of data and materials}

The datasets used and/or analyzed during the current study are available from the corresponding author on reasonable request.

\section{Authors' contributions}

YJ supervised the study; SJ and GL acquired the data and wrote a draft of the manuscript; YJ, CC, GL and YX prepared the experimental materials and performed the animal experiments and in vitro assays; CC, XS and LH interpreted data, performed the statistical analysis and analyzed the resul the ts; YJ and SJ revised and approved the final version of the manuscript. All authors read and approved the manuscript and agree to be accountable for all aspects of the research in ensuring that the accuracy or integrity of any part of the work are appropriately investigated and resolved.

\section{Ethics approval and consent to participate}

All experimental animal protocols were approved by the Institutional Animal Care and Use Committee of Wenzhou Medical University (Wenzhou, Zhejiang, China).

\section{Patient consent for publication}

Not applicable.

\section{Competing interests}

The authors declare that they have no competing interests.

\section{References}

1. Jonas JB, Aung T, Bourne RR, Bron AM, Ritch R and Panda-Jonas S: Glaucoma. Lancet 390: 2183-2193, 2017.

2. Bourne RR, Stevens GA, White RA, Smith JL, Flaxman SR, Price H, Jonas JB, Keeffe J, Leasher J, Naidoo K, et al: Causes of vision loss worldwide, 1990-2010: A systematic analysis. Lancet Glob Health 1: e339-e349, 2013.

3. Stevens GA, White RA, Flaxman SR, Price H, Jonas JB, Keeffe J, Leasher J, Naidoo K, Pesudovs K, Resnikoff S, et al: Global prevalence of vision impairment and blindness: Magnitude and temporal trends, 1990-2010. Ophthalmology 120: 2377-2384, 2013.
4. Doozandeh A and Yazdani S: Neuroprotection in glaucoma. J Ophthalmic Vis Res 11: 209-220, 2016.

5. Tian K, Shibata-Germanos S, Pahlitzsch M and Cordeiro MF: Current perspective of neuroprotection and glaucoma. Clin Ophthalmol 9: 2109-2118, 2015.

6. Calkins DJ, Pekny M, Cooper ML and Benowitz L; Lasker/IRRF Initiative on Astrocytes and Glaucomatous Neurodegeneration Participants: The challenge of regenerative therapies for the optic nerve in glaucoma. Exp Eye Res 157: 28-33, 2017.

7. Bond WS and Rex TS: Evidence that erythropoietin modulates neuroinflammation through differential action on neurons, astrocytes and microglia. Front Immunol 5: 523, 2014.

8. Perez VL and Caspi RR: Immune mechanisms in inflammatory and degenerative eye disease. Trends Immunol 36: 354-363, 2015.

9. Cueva Vargas JL, Belforte N and Di Polo A: The glial cell modulator ibudilast attenuates neuroinflammation and enhances retinal ganglion cell viability in glaucoma through protein kinase A signaling. Neurobiol Dis 93: 156-171, 2016.

10. Xu Y, Yang B, Hu Y, Lu L, Lu X, Wang J, Xu F, Yu S, Huang J and Liang $\mathrm{X}$ : Wogonin prevents TLR4-NF- $\kappa \mathrm{B}$-medicated neuro-inflammation and improves retinal ganglion cells survival in retina after optic nerve crush. Oncotarget 7: 72503-72517, 2016.

11. Lindsey JD, Duong-Polk KX, Hammond D, Leung CK and Weinreb RN: Protection of injured retinal ganglion cell dendrites and unfolded protein response resolution after long-term dietary resveratrol. Neurobiol Aging 36: 1969-1981, 2015.

12. Akyol S, Ugurcu V, Balci M, Gurel A, Erden G, Cakmak O and Akyol O: Caffeic acid phenethyl ester: Its protective role against certain major eye diseases. J Ocul Pharmacol Ther 30: 700-708, 2014.

13. Natarajan K, Singh S, Burke TR Jr, Grunberger D and Aggarwal BB: Caffeic acid phenethyl ester is a potent and specific inhibitor of activation of nuclear transcription factor NF-kappa B. Proc Natl Acad Sci USA 93: 9090-9095, 1996.

14. Parlakpinar H, Ozer MK, Ucar M, Gaffaroglu M, Vardi N, Koc M and Acet A: Protective effects of caffeic acid phenethyl ester (CAPE) on amikacin-induced nephrotoxicity in rats. Cell Biochem Funct 24: 363-367, 2006.

15. Kujumgiev A, Tsvetkova I, Serkedjieva Y, Bankova V, Christov R and Popov S: Antibacterial, antifungal and antiviral activity of propolis of different geographic origin. J Ethnopharmacol 64: 235-240, 1999.

16. Ren J, Zhang N, Liao H, Chen S, Xu L, Li J, Yang Z, Deng W and Tang Q: Caffeic acid phenethyl ester attenuates pathological cardiac hypertrophy by regulation of MEK/ERK signaling pathway in vivo and vitro. Life Sci 181: 53-61, 2017.

17. Russo A, Cardile V, Sanchez F, Troncoso N, Vanella A and Garbarino JA: Chilean propolis: Antioxidant activity and antiproliferative action in human tumor cell lines. Life Sci 76: 545-558, 2004.

18. Tolba MF, Omar HA, Azab SS, Khalifa AE, Abdel-Naim AB and Abdel-Rahman SZ: Caffeic acid phenethyl ester: A review of its antioxidant activity, protective effects against ischemia-reperfusion injury and drug adverse reactions. Crit Rev Food Sci Nutr 56: 2183-2190, 2016.

19. Irmak MK, Fadillioglu E, Sogut S, Erdogan H, Gulec M, Ozer M, Yagmurca M and Gozukara ME: Effects of caffeic acid phenethyl ester and alpha-tocopherol on reperfusion injury in rat brain. Cell Biochem Funct 21: 283-289, 2003.

20. Amodio R, De Ruvo C, Sacchetti A, Di Santo A, Martelli N, Di Matteo V, Lorenzet R, Poggi A, Rotilio D, Cacchio M and Esposito E: Caffeic acid phenethyl ester blocks apoptosis induced by low potassium in cerebellar granule cells. Int J Dev Neurosci 21: 379-389, 2003.

21. Zhong H, Cui L, Xu F, Chen L, Jiang L, Huang H, Xu J, Zhao X, Li L, Zeng S and Li M: Up-regulation of Wipl involves in neuroinflammation of retinal astrocytes after optic nerve crush via NF- $\kappa B$ signaling pathway. Inflamm Res 65: 709-715, 2016.

22. Shi Y, Wu X, Gong Y, Qiu Y, Zhang H, Huang Z and Su K: Protective effects of caffeic acid phenethyl ester on retinal ischemia/reperfusion injury in rats. Curr Eye Res 35: 930-937, 2010.

23. Valapala M, Hose S, Gongora C, Dong L, Wawrousek EF, Samuel Zigler J Jr and Sinha D: Impaired endolysosomal function disrupts Notch signalling in optic nerve astrocytes. Nat Commun 4: 1629, 2013.

24. Mi Hand Barres BA: Purification and characterization of astrocyte precursor cells in the developing rat optic nerve. J Neurosci 19: 1049-1061, 1999.

25. Livak KJ and Schmittgen TD: Analysis of relative gene expression data using real-time quantitative PCR and the 2(-Delta Delta C(T)) method. Methods 25: 402-408, 2001. 
26. Qu J and Jakobs TC: The time course of gene expression during reactive gliosis in the optic nerve. PLoS One 8: e67094, 2013.

27. McKinnon SJ, Schlamp CL and Nickells RW: Mouse models of retinal ganglion cell death and glaucoma. Exp Eye Res 88: 816-824, 2009.

28. Schaub JA, Kimball EC, Steinhart MR, Nguyen C, Pease ME, Oglesby EN, Jefferys JL and Quigley HA: Regional retinal ganglion cell axon loss in a murine glaucoma model. Invest Ophthalmol Vis Sci 58: 2765-2773, 2017.

29. Wisely CE, Sayed JA, Tamez H, Zelinka C, Abdel-Rahman MH, Fischer AJ and Cebulla CM: The chick eye in vision research: An excellent model for the study of ocular disease. Prog Retin Eye Res 61: 72-97, 2017.

30. Rathnasamy G, Foulds WS, Ling EA and Kaur C: Glutamate inhibits the pro-survival effects of insulin-like growth factor-1 on retinal ganglion cells in hypoxic neonatal rat retina. Mol Neurobiol 54: 3453-3464, 2017.

31. Sobrado-Calvo P, Vidal-Sanz M and Villegas-Pérez MP: Rat retinal microglial cells under normal conditions, after optic nerve section, and after optic nerve section and intravitreal injection of trophic factors or macrophage inhibitory factor. J Comp Neurol 501: 866-878, 2007.

32. Bodeutsch N, Siebert H, Dermon C and Thanos S: Unilateral injury to the adult rat optic nerve causes multiple cellular responses in the contralateral site. J Neurobiol 38: 116-128, 1999.

33. Rovere G, Nadal-Nicolás FM, Sobrado-Calvo P, García-Bernal D, Villegas-Pérez MP, Vidal-Sanz M and Agudo-Barriuso M: Topical treatment with bromfenac reduces retinal gliosis and inflammation after optic nerve crush. Invest Ophthalmol Vis Sci 57: 6098-6106, 2016.

34. Li X, Long J, He T, Belshaw R and Scott J: Integrated genomic approaches identify major pathways and upstream regulators in late onset Alzheimer's disease. Sci Rep 5: 12393, 2015.

35. Li Y, Wang LM, Xu JZ, Tian K, Gu CX and Li ZF: Gastrodia elata attenuates inflammatory response by inhibiting the NF- $\kappa \mathrm{B}$ pathway in rheumatoid arthritis fibroblast-like synoviocytes. Biomed Pharmacother 85: 177-181, 2017.

36. Oguiza A, Recio C, Lazaro I, Mallavia B, Blanco J, Egido J and Gomez-Guerrero C: Peptide-based inhibition of $\mathrm{I} \kappa \mathrm{B}$ kinase/nuclear factor- $\kappa \mathrm{B}$ pathway protects against diabetes-associated nephropathy and atherosclerosis in a mouse model of type 1 diabetes. Diabetologia 58: 1656-1667, 2015
37. Saccà SC, Gandolfi S, Bagnis A, Manni G, Damonte G, Traverso CE and Izzotti A6: From DNA damage to functional changes of the trabecular meshwork in aging and glaucoma. Ageing Res Rev 29: 26-41, 2016.

38. Agapova OA, Kaufman PL and Hernandez MR: Androgen receptor and NFKB expression in human normal and glaucomatous optic nerve head astrocytes in vitro and in experimental glaucoma. Exp Eye Res 82: 1053-1059, 2006.

39. Erb C: Importance of the nuclear factor kappaB for the primary open angle glaucoma-a hypothesis. Klin Monbl Augenheilkd 227: 120-127, 2010 (In German).

40. Khan MN,Lane ME, McCarron PA and Tambuwala MM: Caffeic acid phenethyl ester is protective in experimental ulcerative colitis via reduction in levels of pro-inflammatory mediators and enhancement of epithelial barrier function. Inflammopharmacology 26 : 561-569, 2018.

41. Ak H, Gülşen İ, Karaaslan T, Alaca İ, Candan A, Koçak H, Atalay T, Celikbilek A, Demir İ and Yilmaz T: The effects of caffeic acid phenethyl ester on inflammatory cytokines after acute spinal cord injury. Ulus Travma Acil Cerrahi Derg 21: 96-101, 2015.

42. Spaide RF: Retinal vascular cystoid macular edema: Review and new theory. Retina 36: 1823-1842, 2016

43. Tura A, Schuettauf F, Monnier PP, Bartz-Schmidt KU and Henke-Fahle S: Efficacy of Rho-kinase inhibition in promoting cell survival and reducing reactive gliosis in the rodent retina. Invest Ophthalmol Vis Sci 50: 452-461, 2009.

44. Chrysostomou V, Rezania F, Trounce IA and Crowston JG: Oxidative stress and mitochondrial dysfunction in glaucoma. Curr Opin Pharmacol 13: 12-15, 2013.

This work is licensed under a Creative Commons Attribution-NonCommercial-NoDerivatives 4.0 International (CC BY-NC-ND 4.0) License. 